PROCEEDINGS OF THE

AMERICAN MATHEMATICAL SOCIETY

Volume 126, Number 6, June 1998, Pages 1645-1653

S 0002-9939(98)04480-3

\title{
NON-NOETHERIAN REGULAR RINGS OF DIMENSION 2
}

\author{
JAMES J. ZHANG
}

(Communicated by Ken Goodearl)

\begin{abstract}
We study connected, not necessarily noetherian, regular rings of global dimension 2 .
\end{abstract}

\section{INTRODUCTION}

Throughout $k$ is a field and $A$ is a connected $k$-algebra; thus $A=\bigoplus_{n \geq 0} A_{n}$ and $A_{0}=k$. The augmentation ideal is the unique maximal graded ideal $\mathfrak{m}:=$ $\bigoplus_{i>0} A_{i}$. We call $A / \mathfrak{m}$ the trivial module, and usually denote it by $k$. The (left and right) global dimension of $A$ is equal to the projective dimension of $k_{A}$. The algebra $A$ is regular if it has finite global dimension, $d$ say, and

$$
\operatorname{Ext}^{i}(k, A) \cong \begin{cases}k & \text { if } i=d \\ 0 & \text { if } i \neq d\end{cases}
$$

In some papers this is called Artin-Schelter regular. In the definition we do not assume that $A$ is finitely generated or even locally finite, but it is proved in $[9,3.1]$ that a regular ring is finitely generated. Also we do not require $\operatorname{GKdim} A<\infty$ in the definition.

Noetherian regular rings of global dimension no more than 2 are easy to classify. Noetherian regular rings of global dimension 3 are classified and studied in $[1,2,3$, $7,8]$. Here we classify regular rings of global dimension 2 which are not necessarily noetherian.

Theorem 0.1. A connected ring is regular of global dimension 2 if and only if it is isomorphic to the algebra $k\left\langle x_{1}, \cdots, x_{n}\right\rangle /(b)$ satisfying the following conditions:

1. $n \geq 2$;

2. if the $x_{i}$ 's are labeled so that $1 \leq \operatorname{deg} x_{1} \leq \cdots \leq \operatorname{deg} x_{n}$, then $\operatorname{deg} x_{i}+\operatorname{deg} x_{n-i}$ is a constant for all $i$;

3. there is a graded algebra automorphism $\sigma$ of the free algebra $k\left\langle x_{1}, \cdots, x_{n}\right\rangle$ such that $b=\sum_{i=1}^{n} x_{i} \sigma\left(x_{n-i}\right)$.

Theorem 0.2. Let $A$ be an algebra in Theorem 0.1. Then the following hold.

1. A is noetherian if and only if $A$ has finite GK-dimension, if and only if $n=2$.

2. A is a domain. If $n>2$, then it is not an Ore domain.

Received by the editors December 3, 1996.

1991 Mathematics Subject Classification. Primary 16W50, 16E10, 16E70.

Key words and phrases. Connected algebra, global dimension, regular algebra.

This research was supported by an NSF Postdoctoral Fellowship.

(C)1998 American Mathematical Society 
3. For every proper graded subspace $W \subset \bigoplus_{i=1}^{n} k x_{i}$, the subalgebra generated by $W$ is free. As a consequence, if $n>2, A$ does not satisfy a polynomial identity.

4. If $n>2, A$ does not have non-trivial normal elements.

We also study one-relator quadratic algebras. It is well-known that every onerelator quadratic algebra is Koszul and its global dimension is either infinite if the relation is $x^{2}=0$ or 2 otherwise. Here are some other properties.

Theorem 0.3. Let $V$ be a finite dimensional vector space and $A$ a one-relator quadratic algebra $k\langle V\rangle /(b)$ for some $0 \neq b \in V \otimes V$. Then the following hold.

1. $A$ is noetherian if and only if $\operatorname{dim} V=1$ or $\operatorname{dim} V=2$ and $b \neq x y$.

2. $A$ is a domain if and only if $b \neq x y$ for some $x, y \in V$.

3. If $A$ has a non-trivial normal element, then $\operatorname{dim} V=1$ or $\operatorname{dim} V=2$ and $b \neq x y$.

4. If $W \subset V$ is a subspace such that $b \notin W \otimes V$, then the subalgebra $k[W]$ is free.

\section{Regular Rings of GLOBAL Dimension 2}

Let $F=k\left\langle x_{1}, \cdots, x_{n}\right\rangle$ be a connected graded free algebra with augmentation ideal $\mathfrak{m}_{F}$. Let $\left\{y_{1}, \cdots, y_{n}\right\}$ be another minimal graded generating set of $F$, namely, $\left\{\bar{y}_{1}, \cdots, \bar{y}_{n}\right\}$ is a basis of the graded vector space $\mathfrak{m}_{F} / \mathfrak{m}_{F}^{2}$. Suppose that $b=$ $\sum_{i=1}^{r} x_{i} y_{i}$ where $1 \leq r \leq n$ and where $\operatorname{deg} x_{i}+\operatorname{deg} y_{i}=e$ for all $1 \leq i \leq r$. The integer $r$ is called the rank of $b$. Define $A$ to be the connected one-relator algebra $F /(b)$. Since $b \in \mathfrak{m}_{F}^{2}, A$ and $F$ have the same minimal generating sets. We call $F$ the covering free algebra of $A$. For any element $f$ in $F$ we will use the same letter $f$ for the image in $A$. We will use $={ }_{*}$ for equality in the covering free algebra $F$ and use ordinary $=$ for equality in $A$. These one-relator algebras form a special class of what W. Dicks studied in [5]. The Hilbert series of $A$ is defined by

$$
H_{A}(t)=\sum_{i} \operatorname{dim} A_{i} t^{i}
$$

Suppose that $H_{A}(t)=q(t) / p(t)$ for some relatively prime polynomials with integer coefficients and $p(0)=1$ (we will see that this property holds for all algebras studied in this paper). If every root of $p(t)$ has absolute value 1 , then $A$ has finite GelfandKirillov dimension; otherwise $A$ has exponential growth $[9,2.2]$. If $M=\bigoplus_{i \in \mathbb{Z}} M_{i}$ is a graded module, then the $l$-th degree shift of $M$ is $M(l):=\bigoplus_{i \in \mathbb{Z}} M_{i+l}$.

Parts 1 and 2 of the following proposition are well-known. Since we will use these several times later, we include a proof here.

Proposition 1.1. Let $A$ be the connected algebra $k\left\langle x_{1}, \cdots, x_{n}\right\rangle /(b)$ defined above and $r=\operatorname{rank} b$.

1. If $b=a x_{1}^{2}$ for some non-zero scalar $a$, then

(a) $\operatorname{gldim} A=\infty$,

(b) $H_{A}(t)=\left(1+t^{\operatorname{deg} x_{1}}\right)\left[\left(1+t^{\operatorname{deg} x_{1}}\right)\left(1-\sum_{i=1}^{n} t^{\operatorname{deg} x_{i}}\right)+t^{2 \operatorname{deg} x_{1}}\right]^{-1}$.

2. If $r>1$, or if $r=1$ and $x_{1}, y_{1}$ are linearly independent, then

(a) $\operatorname{gldim} A=2$,

(b) $H_{A}(t)=\left(1-\sum_{i=1}^{n} t^{\operatorname{deg} x_{i}}+t^{\operatorname{deg} b}\right)^{-1}$.

3. $A$ is regular if and only if $r=n>1$. 
Proof. 1. Note that $A$ has no non-resolvable ambiguities in the sense of [4] and all monomials not containing $x_{1}^{2}$ form a basis of $A$, whence the right annihilator $r\left(x_{1}\right):=\left\{a \in A \mid x_{1} a=0\right\}$ is equal to $x_{1} A$. By using this property we see that the minimal projective resolution of $k_{A}$ is

$$
\cdots \longrightarrow A(-3 l) \longrightarrow A(-2 l) \longrightarrow \bigoplus_{i} A\left(-l_{i}\right) \longrightarrow A \longrightarrow k \longrightarrow 0
$$

where $l=\operatorname{deg} x_{1}$ and the boundary map from $A(-(p+1) l)$ to $A(-p l)$ is multiplication by $x_{1}$. Hence the statements follow from (1-1).

2. Let $S$ be the idealizer $\mathbb{I}(b F):=\{f \in F \mid f b \in b F\}$. We claim that $S=k+b F$. Suppose that $f b=_{*} b a$ for some $f, a \in \mathfrak{m}_{F}-\{0\}$ and write $f$ as $\sum_{i} x_{i} a_{i}$ in $F$. Then

$$
\begin{array}{ll}
a_{j} b={ }_{*} y_{j} a & \forall j=1, \cdots, r ; \\
a_{j} b={ }_{*} 0 & \forall j=r+1, \cdots, n .
\end{array}
$$

Since $F$ is a domain, $a_{j} \neq_{*} 0$ for $j \leq r$ and $a_{j}=_{*} 0$ for $j \geq r+1$. If $a_{j_{0}} \in k^{\times}$ for some $j_{0} \leq r$, then (1-2) shows that $y_{j_{0}}\left(a_{j_{0}}^{-1} a\right)=_{*} b=_{*} \sum_{i=1}^{r} x_{i} y_{i}$. This cannot happen in the free algebra $F$ when either $r \geq 2$ or $r=1$ and $\left\{x_{1}, y_{1}\right\}$ are linearly independent. Thus we obtain a contradiction and hence $a_{i} \in \mathfrak{m}$ for all $j \leq r$. Since $\left\{y_{1}, \cdots, y_{n}\right\}$ is a minimal generating set of $F,(1-2)$ implies that $a_{j}=_{*} y_{j} g_{j}$, so $g_{j} b={ }_{*} a$ by factoring out $y_{j}$ from (1-2) for all $j \leq r$. Since $F$ is a domain, $g_{j}={ }_{*} g_{1}$ and hence

$$
f=* \sum_{i=1}^{n} x_{i} a_{i}=* \sum_{j=1}^{r} x_{j} a_{j}=* \sum_{j=1}^{r} x_{j} y_{j} g_{1}={ }_{*} b g_{1} .
$$

Thus $S=k+b F$ and the eigenring (defined in [5, 2.1]) is $E:=S / b F \cong k$. By $[5$, 5.3], gldim $A=\operatorname{gldim} E+2=2$ and by $[5,3.5] H_{A}(t)=\left(1-\sum_{i=1}^{n} t^{\operatorname{deg} x_{i}}+t^{\operatorname{deg} b}\right)^{-1}$.

3 . We only need to consider the algebras in part 2 . The minimal projective resolution of $k_{A}$ is of the form

$$
0 \longrightarrow A(-e) \longrightarrow \bigoplus A\left(-l_{i}\right) \longrightarrow A \longrightarrow k \longrightarrow 0
$$

where $e=\operatorname{deg} b$ and $l_{i}=\operatorname{deg} x_{i}$ for all $i$. The boundary map $\partial_{1}: \bigoplus A\left(-l_{i}\right) \longrightarrow A$ sends $\left(a_{1}, \cdots, a_{n}\right)$ to $\sum_{i=1}^{n} x_{i} a_{i}$ and the boundary map $\partial_{2}: A(-e) \longrightarrow \bigoplus A\left(-l_{i}\right)$ sends $a$ to $\left(y_{1} a, \cdots, y_{r} a, 0, \cdots, 0\right)$. By definition, $A$ is regular if and only if the dual of $(1-4), \operatorname{Hom}((1-4), A)$ :

$$
0 \longleftarrow A(e) \longleftarrow \bigoplus A\left(l_{i}\right) \longleftarrow A \longleftarrow 0
$$

is the minimal projective resolution of ${ }_{A} k(e)$. The boundary map from $\bigoplus A\left(l_{i}\right)$ to $A(e)$ sends $\left(a_{1}, \cdots, a_{n}\right)$ to $\sum_{i=1}^{r} a_{i} y_{i}$. Hence $A$ is regular if and only if $\left\{y_{i}\right\}_{i=1}^{r}$ is a minimal generating set of $A$, if and only if $r=n$.

Proof of Theorem 0.1. Suppose that 1, 2, 3 hold. Let $y_{i}=\sigma\left(x_{n-i}\right)$. Then $A$ is regular of global dimension 2 by Proposition 1.1.3.

Conversely suppose that $A$ is connected and regular of global dimension two. By $[9,3.1 .1]$, we have a minimal free resolution of $k_{A}$

$$
0 \longrightarrow A(-e) \longrightarrow \bigoplus_{i=1}^{n} A\left(-l_{i}\right) \longrightarrow A \longrightarrow k \longrightarrow 0
$$


where $n$ is finite. The Hilbert series is $H_{A}(t)=\left(1-\sum_{i} t^{l_{i}}+t^{e}\right)^{-1}$. We may assume that $l_{1} \leq l_{2} \leq \cdots \leq l_{n}$. If $n=1$, we obtain an algebra with infinite global dimension (Proposition 1.1.1). Hence $n \geq 2$. By [9, 3.1.4],

$$
t^{e}\left(1-\sum_{i} t^{-l_{i}}+t^{-e}\right)=\left(1-\sum_{i} t^{l_{i}}+t^{e}\right),
$$

whence $l_{i}+l_{n-i}=e$ for all $i=1, \cdots, n$. The boundary map from $\bigoplus_{i=1}^{n} A\left(-l_{i}\right)$ to $A$ sends $\left(a_{1}, \cdots, a_{n}\right)$ to $\sum_{i} x_{i} a_{i}$ where $\left\{x_{i}\right\}$ is the minimal set of generators and $\operatorname{deg} x_{i}=l_{i}$, and the boundary map from $A(-e)$ to $\bigoplus_{i=1}^{n} A\left(-l_{i}\right)$ sends $a$ to $\left(y_{1} a, \cdots y_{n} a\right)$ for some $y_{i} \in \mathfrak{m}$. Consequently, $A$ has one relation of degree $e$, of form $x_{1} y_{1}+x_{2} y_{2}+\cdots x_{n} y_{n}=0$. Since $A$ is regular, the dual of (1-6) (see (1-5)) is the projective resolution of ${ }_{A} k(e)$, so $\left\{y_{i}\right\}_{i=1}^{n}$ is a minimal generating set of $A$. Now any homogeneous minimal generating set for $A$ is also a minimal generated set for $F$. Thus $F=k\left\langle y_{1}, \cdots, y_{n}\right\rangle$ and $\sigma: x_{i} \longrightarrow y_{n-i}$ defines a graded algebra automorphism of $F$. Since the relation is homogeneous, we have $\operatorname{deg} y_{i}=e-l_{i}=l_{n-i}$ for all $i$. Therefore $A$ is isomorphic to $F /(b)$ and 1, 2, 3 of Theorem 0.1 hold.

By Theorem 0.1.2, if $A$ is generated by three elements $x_{1}, x_{2}, x_{3}$ with $\operatorname{deg} x_{1} \leq$ $\operatorname{deg} x_{2} \leq \operatorname{deg} x_{3}$ and $\operatorname{deg} x_{1}+\operatorname{deg} x_{3} \neq 2 \operatorname{deg} x_{2}$, then $A$ is not regular of global dimension two. Noetherian regular rings of global dimension 2 are easily determined and that is the case when $n=2$ in Theorem 0.1. The following Corollary is part 1 of Theorem 0.2 .

Corollary 1.2. Let $A$ be a connected regular ring of global dimension 2, generated by $n$ elements (see Theorem 0.1). Then the following statements are equivalent.

1. $n=2$.

2. A is noetherian.

3. $\operatorname{GKdim}(A)=2$.

4. $\operatorname{GKdim}(A)<\infty$.

Proof. If $A$ is in Theorem 0.1 and $n=2$, then it is easy to see that $A$ is noetherian of GK-dimension $2[9,3.5]$.

If $A$ is in Theorem 0.1 (or even in Proposition 1.1) and $n>2$, then it has exponential growth, and by $[9,1.2]$, it is neither left nor right noetherian.

Next we prove other parts of Theorem 0.2. For simplicity, we use $\bar{f}$ for the image of $f$ in $A / \mathfrak{m}^{2}$ for all $f \in A$.

Proposition 1.3. Let $A$ be regular of global dimension 2, generated by $n$ elements. Then the following statements hold.

1. A is a domain.

2. Suppose that $n>2$. Let $x, f$ and $g$ be homogeneous elements in $\mathfrak{m}-\{0\}$ and suppose that $x \notin \mathfrak{m}^{2}$. If $f g \in x A$ ( $g f \in A x$ respectively), then $f \in x A$ $(f \in A x$ respectively).

Remark 1.4. We do not have any example of a noetherian or non-noetherian regular ring which is not a domain.

Proof. If $A$ is a regular ring of global dimension 2 and $n=2$, then it is described in $[9,3.3]$, and in particular, it is a domain. In the rest of the proof we assume that $n>2$. 
Since $A$ is $\mathbb{N}$-graded, $A$ being a graded domain implies that $A$ is a domain. Pick a minimal generating set $\left\{x_{i} \mid i=1, \cdots, n\right\}$ such that $x=x_{n}$. Hence it suffices to show the following statement:

- Let $f$ and $g$ be homogeneous elements in $\mathfrak{m}-\{0\}$. Then

(a) $f g \neq 0$

(b) $f g \in x_{n} A$ implies $f \in x_{n} A$.

We will prove $(\bullet)$ by induction on $m:=\operatorname{deg} f+\operatorname{deg} g$. Nothing needs to be proved when $m=1$. Now suppose that $m>1$ and assume that the statement $(\bullet)$ holds for all cases when $\operatorname{deg} f+\operatorname{deg} g<m$.

Case 1: $m<e$.

Since $\bigoplus_{i<e} A_{i}=\bigoplus_{i<e} F_{i}$, it suffices to show that (a) and (b) hold in $F$. Hence (a) follows because $F$ is a domain and (b) follows because $x_{n}$ is in a minimal generating set of $F$.

Case 2: $m=e$.

(a) If $f g=0$ in $A$, then $f g=_{*} l b$. Since $f g \neq_{*} 0, l \neq 0$. Write $f={ }_{*} \sum_{i} x_{i} a_{i}$; we have $a_{i} g=_{*} l y_{i}$ for all $i$. Passing to $\mathfrak{m} / \mathfrak{m}^{2}$, we have $\bar{y}_{i} \in k \bar{g}$ for all $i$. This contradicts the fact that $\left\{\bar{y}_{i}\right\}_{i=1}^{n}$ are linearly independent. Therefore $f g \neq 0$.

(b) If $f g \in x_{n} A$, then $f g={ }_{*} l b+x_{n} c$ for $l \in k$ and for some $c \in F$. By expanding $f$ we have $a_{i} g={ }_{*} l y_{i}$ for $i<n$ and $a_{n} g={ }_{*} l y_{n}+c$. If $l \neq 0$, then this contradicts the fact $\left\{\bar{y}_{i}\right\}_{i<n}$ are linearly independent. Hence $l=0$, whence $a_{i}={ }_{*} 0$ for all $i<n$. Therefore $f=x_{n} a_{n} \in x_{n} A$.

Case 3: $m>e$.

(a) If $f g=0$, then, in $F$, we have

$$
f g=_{*} \sum_{j=1}^{q} f_{j} b g_{j}+f_{0} b+b g_{-1}
$$

where $f_{i}, g_{i} \in \mathfrak{m}_{F}$. We always assume each term in the equation has degree $m$. Write $f_{j}=* \sum_{i} x_{i} f_{i j}$ and $f=* \sum_{i} x_{i} a_{i}$; we obtain

$$
a_{i} g={ }_{*} f_{j} f_{i j} b g_{j}+f_{i 0} b+y_{i} g_{-1} \forall i
$$

For every $i, a_{i} g=y_{i} g_{-1} \in y_{i} A$. If $g_{-1}=0$, then by induction hypothesis (a), $a_{i}=0$ for all $i$, so $f=0$, a contradiction. Hence $g_{-1} \neq 0$, so $y_{i} g_{-1} \neq 0$ by induction hypothesis (a). This implies that $a_{i} \neq 0$ for all $i$. We claim that $a_{i} \in \mathfrak{m}$ $(*)$. If not, let $a_{i_{0}} \in k^{\times}$. Thus $g=z_{i_{0}} g_{-1}$ where $z_{i_{0}}=y_{i_{0}} a_{i_{0}}^{-1} \in \mathfrak{m}-\mathfrak{m}^{2}$. For each $i, a_{i} z_{i_{0}} g_{-1}=y_{i} g_{-1}$. By induction hypothesis (a), $a_{i} z_{i_{0}}=y_{i}$ and hence $\bar{y}_{i} \in k \bar{z}_{i_{0}}$ for all $i$. This contradicts the fact that $\left\{\bar{y}_{i}\right\}_{i=1}^{n}$ are linearly independent. Thus we proved our claim $(*)$. By induction hypothesis (b), $a_{i}=y_{i} w_{i}$. Factoring out $a_{i}$ from $a_{i} g=y_{i} g_{-1}$ (by using induction hypothesis (a)), we obtain $w_{i} g=g_{-1}$ for all $i$. By induction hypothesis (a), $w_{i}=w_{1}$ and hence $f=\sum_{i} x_{i} y_{i} w_{1}=b w_{1}=0$. This contradicts $f \neq 0$ in $A$, and hence (a) follows.

(b) If $0 \neq f g \in x_{n} A$, then $f g=x_{n} c$ for some $c \neq 0$ with $\operatorname{deg} c=m-\operatorname{deg} x_{n}>0$. Similar to (1-7) we have

$$
f g={ }_{*} \sum_{j=1}^{n} f_{j} b g_{j}+f_{0} b+b g_{-1}+x_{n} c
$$


and similar to (1-8), we have

$$
a_{i} g={ }_{*} \sum_{j} f_{i j} b g_{j}+f_{i 0} b+y_{i} g_{-1} \forall i<n .
$$

Hence $a_{i} g=y_{i} g_{-1} \in y_{i} A$ for all $i<n$. If $g_{-1}=0$, then by induction hypothesis (a) $a_{i}=0$ for all $i$ and then $f=x_{n} a_{n} \in x_{n} A$. Now we suppose that $g_{-1} \neq 0$. Similar to $(*)$, we may assume $a_{i} \in \mathfrak{m}$ for all $i<n$ (in this case we use the fact that $\left\{\bar{y}_{i}\right\}_{i<n}$ are linearly independent). Since $y_{i} \in \mathfrak{m}-\mathfrak{m}^{2}$, by induction hypothesis (b), $a_{i}=y_{i} w_{i}$ for all $i<n$. Factoring out $y_{i}$ from $a_{i} g=y_{i} g_{-1}$ we obtain that $w_{i} g=g_{-1}$ for all $i<n$. By induction hypothesis (a), $w_{i}=w_{1}$ for all $i<n$, and hence

$$
\begin{aligned}
f=\sum_{i} x_{i} a_{i} & =x_{n} a_{n}+\sum_{i<n} x_{i} a_{i}=x_{n} a_{n}+\sum_{i<n} x_{i} y_{i} w_{1} \\
& =x_{n} a_{n}+\left(b-x_{n} y_{n}\right) w_{1}=x_{n}\left(a_{n}-y_{n} w_{1}\right) \in x_{n} A .
\end{aligned}
$$

Thus (b) follows.

Proposition 1.5. Let $A$ be a one-relator algebra $k\left\langle x_{1}, \cdots, x_{n}\right\rangle /(b)$ where $b=$ $\sum_{i} x_{i} z_{i}$. Suppose that, for some $s, z_{s}$ is a right regular element. Then $\sum_{i \neq s} x_{i} A=$ $\bigoplus_{i \neq s} A_{i}$ and hence the subalgebra generated by $\left\{x_{i} \mid i \neq s\right\}$ is free.

As a consequence, if $A$ is a regular algebra of global dimension 2 and $\left\{x_{i} \mid 1 \leq\right.$ $i \leq n\}$ is a minimal generating set of $A$, then the subalgebra $k\left[x_{i} \mid i \neq s\right]$ is free for any $s$.

Proof. It suffices to show that $\sum_{i \neq s} x_{i} a_{i}=0$ implies $a_{i}=0$ in $A$ for all $i \neq s$. Suppose that $\sum_{i \neq s} x_{i} a_{i}=0$ for some $a_{i} \in A$. As the proof of Proposition 1.3, there are $f_{j}$ and $g_{j}$ with $f_{j} \in \mathfrak{m}_{F}$ such that

$$
\sum_{i \neq s} x_{i} a_{i}=* \sum_{j=0}^{q} f_{j} b g_{j}+b g_{-1}=* \sum_{j=0}^{q} \sum_{i=1}^{n} x_{i} f_{i j} b g_{j}+\sum_{i=1}^{n} x_{i} z_{i} g_{-1},
$$

and hence

$$
\left(1-\delta_{i s}\right) a_{i}=z_{i} g_{-1} \quad \text { in } \quad A .
$$

If $i=s$, then $z_{s} g_{-1}=0$, so $g_{-1}=0$ because $z_{s}$ is right regular. If $i \neq s$, then $a_{i}=z_{i} g_{-1}=0$.

If $A$ is regular algebra, then $A$ is a domain [Proposition 1.3] and hence $z_{s}$ is a regular element. Therefore the statement follows.

The proofs of parts 3 and 4 of the following are the same as ones of $[11,2.3]$ which are stated only for quadratic algebras.

Proposition 1.6. Let $A$ be a connected algebra and $x$ a homogeneous element in $\mathfrak{m}-\{0\}$ satisfying the following two conditions:

(a) $x$ is right regular and $x A \neq \mathfrak{m}$;

(b) if $f$ and $g$ are in $\mathfrak{m}-\{0\}$ and $f g \in x A$, then $f \in x A$.

Then the following statements hold.

1. A is a domain.

2. $A$ is not an Ore domain and it does not satisfy a polynomial identity.

3. If $a, y$ are in $A-\{0\}$, then $x a=$ ay implies that $x=y$ and $a=l x^{n}$ for some $n \geq 0$ and $l \in k^{\times}$.

4. A has no non-trivial normal elements. 
Proof. 1. Let $f, g$ be non-zero elements in $A$. If $f g=0$, then $f, g \notin k$ and $f g \in x A$. By (b) $f=x f^{\prime}$ and $x f^{\prime} g=0$. By (a), $f^{\prime} g=0$. By induction on $\operatorname{deg} f^{\prime}$, either $f^{\prime}=0$ or $g=0$, a contradiction.

2. Pick $z \in \mathfrak{m}-x A$, by (b), $x A \cap z A=\{0\}$. Hence $A$ is not Ore. Also the subalgebra $k[x, z]$ is free, hence $A$ is not PI.

3. If $x a=a y$ and $a \notin k$, then $a y \in x A$, and by (b), $a=x a^{\prime}$. Hence $x x a^{\prime}=x a^{\prime} y$, and by (a), $x a^{\prime}=a^{\prime} y$. The statement follows from induction on $\operatorname{deg} a$.

4. If $A$ has a non-trivial normal element, then $A$ has a homogeneous normal element of positive degree, say $a$. Hence $x a=a y$ for some $y \in \mathfrak{m}$. By part 3, $x=y$ and $a=l x^{n}$. Thus $x^{n}$ is normal. By (a), there is $z \in \mathfrak{m}-x A$ and by (b) $x A \cap z A=\{0\}$. Therefore $z x^{n} \neq x^{n} c$ for all $c \in A$ and thus $x^{n}$ is not normal, a contradiction.

Parts 2, 3, 4 of Theorem 0.2 follow easily from Propositions 1.3, 1.5 and 1.6.

\section{One-Relator QuAdratic Algebras}

In this section we will apply results from section 1 and results in [11] to onerelator quadratic algebras. From now on $V$ is a vector space with a basis $\left\{x_{i}\right\}_{i=1}^{n}$ and $A$ is a one-relator quadratic algebra $k\langle V\rangle /(b)$ where $b=\sum_{i j} l_{i j} x_{i} x_{j}$ for some $l_{i j} \in k$. It is well-known that $A$ is Koszul and has global dimension 2 or infinity. The rank of the relation $b$ is defined to be the rank of the matrix $\left(l_{i j}\right) \in M_{n}(k)$. This definition of rank coincides with the definition given in section 1 when we consider quadratic algebras. It is easy to see that the rank of $b$ is independent of the choices of the basis of $V$. If $r=\operatorname{rank} b$, then there are two bases $\left\{z_{i}\right\}$ and $\left\{y_{i}\right\}$ of $V$ such that $b=\sum_{i \leq r} z_{i} y_{i}$. Let ${ }_{b} V$ (respectively $V_{b}$ ) denote the subspace generated by $\left\{z_{i} \mid i \leq r\right\}$ (respectively $\left\{y_{i} \mid i \leq r\right\}$ ). Then ${ }_{b} V$ and $V_{b}$ are only dependent on $b$. If $r=1$, then $b=x_{1}^{2}$ or $b=x_{1} x_{2}$ after changing a basis.

Proposition 2.1. Let $A$ be the algebra $k\langle V\rangle /(b)$ and suppose that $b=x_{1} x_{s}$ where $s$ is either 1 or 2 . Then the following statements hold.

1. If $f, g \neq 0$ are in $\mathfrak{m}$, and $f g=0$, then $f \in A x_{1}$ and $g \in x_{s} A$.

2. $\sum_{i>1} x_{i} A=\bigoplus_{i>1} x_{i} A$.

3. The subalgebra generated by $\left\{x_{i} \mid i>1\right\}$ is free.

4. If $j>1$ and $a, y$ are in $A-\{0\}$, then $x_{j} a=$ ay implies that $x_{j}=y$ and $a=l x_{j}^{t}$ for some $l \in k^{\times}$and $t \geq 0$.

5. If $n>1$, then $A$ has no non-trivial normal elements.

Proof. Since $A$ has no non-resolvable ambiguities, all monomials not containing $x_{1} x_{s}$ form a basis of $A$. In particular, $x_{i}$ are right regular for all $i>1$. Let $I$ denote $\left(i_{1}, \cdots, i_{t}\right)$. For every element $a \in F$, write $a=\sum_{I} l_{I} x_{i_{1}} \cdots x_{i_{t}}$ where $\left(i_{p}, i_{p+1}\right) \neq(1, s)$ for all $p$. Define the support of $a$ to be $\operatorname{Supp}(a):=\left\{I \mid l_{I} \neq 0\right\}$. Part 1 and part 2 can be checked easily by expressing elements as sum of monomials, part 3 is a consequence of 2 .

4. As in the proof of Proposition 1.6.3, it suffices to show $a=x_{j} a^{\prime}$. Suppose that this is not true. Then $a=x_{j} a^{\prime}+a_{0}$ where $a_{0}=\sum_{i_{1} \neq j} l_{I} x_{i_{1}} \cdots x_{i_{t}} \neq 0$. Since $x_{j}$ is right regular, $x_{j} a=\sum_{I \in \operatorname{Supp}(a)} l_{I} x_{j} x_{i_{1}} \cdots x_{i_{t}}$. Write $y=\sum_{w} h_{w} x_{w}$; then we have $a y=\sum_{\left(i_{t}, w\right) \neq(1, s)} l_{I} h_{t} x_{i_{1}} \cdots x_{i_{t}} x_{w}$. Comparing the monomials in $x_{j} a$ and ay, we obtain that, if $I=\left(i_{1}, \cdots, i_{t}\right) \in \operatorname{Supp}\left(a_{0}\right)$, then $h_{w} x_{i_{1}} \cdots x_{i_{t}} x_{w}=0$ for all $w$. Thus (i) $h_{w}=0$ for all $w \neq s$ and $h_{s} \neq 0$ and (ii) $i_{t}=1$. As a consequence 
$a y=\left(x_{j} a^{\prime}+a_{0}\right) h_{s} x_{s}=h_{s} x_{j} a^{\prime} x_{s}$. Therefore

$$
|\operatorname{Supp}(a y)|=\left|\operatorname{Supp}\left(a^{\prime}\right)\right|<|\operatorname{Supp}(a)|=\left|\operatorname{Supp}\left(x_{j} a\right)\right|=|\operatorname{Supp}(a y)|,
$$

a contradiction. Therefore part 4 follows.

5. If $A$ has a non-trivial normal element, then $A$ has a homogeneous normal element of positive degree, say $a$. Let $j>1$. Then $x_{j} a=a y$ for some $y$ of degree 1. By part $4, x_{j}=y$ and $a=l x_{j}^{t}$ for some $l \neq 0$ and $t>0$. Hence $x_{j}^{t}$ is normal. But $x_{j}^{t} x_{1} \neq z x_{j}^{t}$ for any $z$, so a contradiction.

Now we are ready to prove Theorem 0.3 .

Proof. 1. If $n=1$, then $A$ is $k[x] /\left(x^{2}\right)$, which is noetherian. If $n=r=2$, then $A$ is a noetherian regular algebra of global dimension two (Corollary 1.2). If $n=2$ and $r=1$, then $A$ is either $k\langle x, y\rangle /\left(x^{2}\right)$ or $k\langle x, y\rangle /(x y)$; neither is noetherian. If $n>2$, then by Proposition 1.1.1(b) and 2(b), $A$ has exponential growth and hence it is not noetherian by $[9,1.2]$.

2 and 3 . We discuss three cases.

Case 1: $r=\operatorname{rank} b>2$. Let $\Phi$ be the set of all subspaces of dimension no more than rank $b-2$. Then by the proof of $[11,0.1(2)]$ we can apply $[11,2.2]$ to $A$ and this $\Phi$. Hence $A$ is a domain and the following statement holds:

(**) If $f$ and $g$ are homogeneous elements in $\mathfrak{m}-\{0\}$ and if $f g \in x A$ for some $x \in V-\{0\}$, then $f \in x A$.

By Proposition 1.6.4, $A$ has no non-trivial normal elements.

Case 2: $r=2$ and ${ }_{b} V \neq V_{b}$. By [11, 2.2 and 2.3], $A$ is a domain and the statement $(* *)$ holds for $x \notin V_{b}$ (in this case $\Phi$ is the set of 1-dimensional subspaces not contained in $V_{b}$ ). By Proposition 1.6.4, $A$ has no non-trivial normal elements.

Case 3: $r=2$ and ${ }_{b} V=V_{b}$. In this case $b=\left(l_{11} x_{1}+l_{21} x_{2}\right) x_{1}+\left(l_{12} x_{1}+l_{22} x_{2}\right) x_{2}$ for some invertible matrix $\left(l_{i j}\right)_{2 \times 2}$. Hence $A=k\langle V\rangle /(b)$ is isomorphic to a twisted algebra $B^{\sigma}$ where $B=k\langle V\rangle /\left(x_{1} x_{2}-x_{2} x_{1}\right)$ and $\sigma: x_{1} \rightarrow l_{12} x_{1}+l_{22} x_{2}, x_{2} \rightarrow$ $-\left(l_{11} x_{1}+l_{21} x_{2}\right), x_{i} \rightarrow x_{i}, \forall i>2$. It is easy to see that the one-relator semi-group $G:=\left\langle x_{1}, \cdots, x_{n} \mid x_{1} x_{2}=x_{2} x_{1}\right\rangle$ is ordered and $B$ is the semi-group algebra $k G$. Therefore $B$ is a domain [6, Prop. A.II.1.4] and hence $A \cong B^{\sigma}$ is a domain [10, 5.2]. Now let $x \in V-{ }_{b} V$ and $f, g \in \mathfrak{m}-\{0\}$. We may assume $x=x_{n}$ and $n>2$. If $f g \in x_{n} A$, then $f g=x_{n} c$ and we have

$$
\sum_{i} x_{i} a_{i} g=* \sum_{i} x_{i} f_{i j} b g_{i}+\sum_{i=1}^{2} x_{i} y_{i} g_{-1}+x_{n} c
$$

in $k\langle V\rangle$, where $f=\sum_{i} x_{i} a_{i}$. Comparing the coefficients in $x_{n}$, we have $a_{n} g=_{*}$ $\sum_{j} f_{n j} b g_{j}+c$ and so $c=a_{n} g$ in $A$. Hence $f g=x_{n} c=x_{n} a_{n} g$. Since $A$ is a domain, $f=x_{n} a_{n} \in x_{n} A$. Again we proved (**) for $x \notin V_{b}$. By Proposition 1.6.4, $A$ has no non-trivial normal elements if $V_{b} \neq V$.

Combining these cases and Proposition 2.1 we prove 2 and 3.

4. If $b \notin W \otimes V$, then there is a subspace $W^{\prime} \supset W$ of dimension $n-1$ such that $b \notin W^{\prime} \otimes V$. So we may assume $\operatorname{dim} W=n-1$. Hence this is a consequence of Proposition 1.5 and part 2 when $r \geq 2$. If $b=x_{1}^{2}$, this is Proposition 2.1.2. It remains to consider the case when $b=x_{1} x_{2}$. By changing a basis we may assume $b=x_{1} y, W=\sum_{i>1} k x_{i}$ and $y \notin k x_{1}$. By Proposition 1.5, it suffices to show that $y$ is a right regular element, which follows from Proposition 2.1.1. 


\section{ACKNOWLEDGMENT}

The author would like to thank S. P. Smith for several discussions on the subject.

\section{REFERENCES}

1. M. Artin and W. Schelter, Graded algebras of global dimension 3, Adv. Math., 66(1987) 171-216. MR 88k:16003

2. M. Artin, J. Tate and M. van den Bergh, Some algebras related to automorphisms of elliptic curves, The Grothendieck Festschrift, Vol. 1, 33-85, Birkhauser, Boston 1990. MR 92e:14002

3. M. Artin, J. Tate and M. van den Bergh, Modules over regular algebras of dimension 3, Invent. Math., 106 (1991) 335-388. MR 93e:16055

4. G. Bergman, The Diamond lemma for ring theory, Adv. Math., 29(1978), 178-216. MR 81b:16001

5. W. Dicks, On the cohomology of one-relator associative algebras, J. Algebra, 97 (1985), 79100. MR 87h: 16041

6. C. Năstăsescu and F. Van Oystaeyen, Graded Ring Theory, North Holland, Amsterdam, 1982. MR 84i: 16002

7. D. R. Stephenson, Artin-Schelter regular algebras of global dimension three, J. Algebra, 183 (1996), no. 1, 55-73. MR 97h:16053

8. D. R. Stephenson, Algebras associated to elliptic curves, Trans. Amer. Math. Soc., 349 (1997), 2317-2340. CMP 97:09

9. D. R. Stephenson and J. J. Zhang, Growth of graded noetherian rings, Proc. Amer. Math. Soc., 125 (1997), 1593-1605. MR 97g:16033

10. J. J. Zhang, Twisted graded algebras and equivalences of graded categories, Proc. London Math. Soc., (3)72 (1996), 281-311. MR 96k:16078

11. J. J. Zhang, Quadratic algebras with few relations, Glasgow Math. J., to appear.

Department of Mathematics, Box 354350, University of Washington, Seattle, WashINGTON 98195

E-mail address: zhang@math.washington.edu 